\title{
Citric Acid Water as an Alternative to Water Restriction for High-Yield Mouse Behavior
}

\author{
(D)Anne E. Urai, ${ }^{1,3}$ Valeria Aguillon-Rodriguez, ${ }^{1}$ 이ês C. Laranjeira, ${ }^{2}$ 'Panny Cazettes, ${ }^{2}$ \\ (D)Zachary F. Mainen, ${ }^{2}$ and ${ }^{\circledR}$ Anne K. Churchland ${ }^{1,4}$
}

https://doi.org/10.1523/ENEURO.0230-20.2020

${ }^{1}$ Cold Spring Harbor Laboratory, Cold Spring Harbor, NY 11724, ${ }^{2}$ Champalimaud Centre for the Unknown, Lisbon, Portugal 1400-038, ${ }^{3}$ Cognitive Psychology Unit, Institute of Psychology and Leiden Institute for Brain and Cognition, Leiden University, 2300 RC Leiden, The Netherlands, and ${ }^{4}$ David Geffen School of Medicine,University of California Los Angeles, Los Angeles, CA 90095

\begin{abstract}
Powerful neural measurement and perturbation tools have positioned mice as an ideal species for probing the neural circuit mechanisms of cognition. Crucial to this success is the ability to motivate animals to perform specific behaviors. One successful strategy is to restrict their water intake, rewarding them with water during a behavioral task. However, water restriction requires rigorous monitoring of animals' health and hydration status and can be challenging for some mice. We present an alternative that allows mice more control over their water intake: free home-cage access to water, made slightly sour by a small amount of citric acid (CA). In a previous study, rats with free access to CA water readily performed a behavioral task for water rewards, although completing fewer trials than under water restriction (Reinagel, 2018). We here extend this approach to mice and confirm its robustness across multiple laboratories. Mice reduced their intake of CA water while maintaining healthy weights. Continuous home-cage access to CA water only subtly impacted their willingness to perform a decision-making task, in which they were rewarded with sweetened water. When free CA water was used instead of water restriction only on weekends, learning and decision-making behavior were unaffected. CA water is thus a promising alternative to water restriction, allowing animals more control over their water intake without interfering with behavioral performance.
\end{abstract}

Key words: citric acid water; decision-making; mouse behavior; water restriction

\section{Significance Statement}

High-throughput, reliable behavioral training is a key requirement for the use of mice in behavioral and systems neuroscience but depends crucially on ability to motivate animals to perform specific behaviors. Here, we present an alternative method to commonly used methods of water restriction: free home-cage access to water, made slightly sour by a small amount of citric acid (CA). This non-labor-intensive, low-error option benefits animal health without hindering behavioral training progress. CA water can serve as a reliable and standardized strategy to achieve high quality task behavior, further facilitating the use of mice in highthroughput behavioral studies.

\section{Introduction}

The mouse is an indispensable species for systems neuroscience, thanks to a rich set of available tools to record and manipulate brain structure and function,

Received May 11, 2020; accepted November 17, 2020; First published January 11, 2021.

The authors declare no competing financial interests. combined with the knowledge of teaching mice specific behavioral tasks. While long thought to be beyond the capacities of the mouse (Abbott, 2010), mice are now

Author contributions: A.E.U., V.A.-R., I.C.L., and F.C. designed research; A.E.U., V.A.-R., I.C.L., and F.C. performed research; I.B.L., Z.F.M., and A.K.C. contributed unpublished reagents/analytic tools; A.E.U. and I.C.L. analyzed data; A.E.U. wrote the paper. Please see the Contribution Table for additional details. 
routinely trained to perform abstract sensory, navigational and decision-making tasks (Carandini and Churchland, 2013; Guo et al., 2014; Goltstein et al., 2018). Crucial to this success is the ability to motivate them to perform specific behaviors.

One successful strategy is to restrict animals' water access, and reward them with fluids for performing behavioral tasks (Skinner, 1936; Toth and Gardiner, 2000; Guo et al., 2014; Goltstein et al., 2018). The mouse's ability to thrive on little water matches its evolutionary past on steppes and other dry environments (Fertig and Edmonds, 1969). In a laboratory setting, however, water restriction requires rigorous monitoring of animal's health and hydration status, usually with daily weighing and precisely measured water intake (Toth and Gardiner, 2000).

We here consider a complementary, alternative approach: free access to water, in which a small amount of citric acid (CA) has been dissolved. CA is a food preservative which makes water taste slightly sour. Rats reduce their intake of CA water without getting sick or dehydrated (Watson et al., 1986). Moreover, free CA water has only subtle impacts on rats' willingness to perform behavioral tasks in which they are rewarded with plain water (Reinagel, 2018). This strategy has significant advantages for both animal welfare and scientific throughput. However, free access to $2 \%$ CA water led to a reduction in trial yield of around 30\% in 2-h daily training sessions (Reinagel, 2018), impeding its use in high-throughput behavioral paradigms. Moreover, it is not known whether this approach would also work in mice. We here set out to test the safety and efficacy of CA water in mice, and explore further strategies that combine the benefits of freely accessible CA water with high trial yields.

Mice readily consumed CA water without adverse health effects. In adult mice, access to 2\% CA water resulted in stable weights, and water intake similar to commonly used water restriction regimes. Giving mice free access to CA water on weekends did not affect trial numbers or task performance on subsequent training days. Specifically, mice with free access to CA water on weekends were motivated to perform many trials (500-1000 daily) evenly over the week, doing a decision-making task in which they earned sugar water. In a large dataset of mouse decision-making behavior (The International Brain

A.E.U. is supported by the German National Academy of Sciences Leopoldina. F.C. was supported by a long-term fellowship (ALTF 461-2016) from the European Molecular Biology Organization and an AXA postdoctoral fellowship. The International Brain Laboratory is supported by the Simons Collaboration on the Global Brain and the Wellcome Trust Grants 209558 and 216324.

Acknowledgements: We thank all members of The International Brain Laboratory behavior working group for support and advice and Peter Dayan, Matteo Carandini, Sonja Hofer, Nick Steinmetz, and Hannah Bayer for valuable comments on this manuscript. Shan Shen provided support with DataJoint queries and Alexander Hastava and Anup Khanal assisted with the preparation of CA HydroGels and mouse weighing.

Correspondence should be addressed to Anne E. Urai at anne.urai@gmail.com.

https://doi.org/10.1523/ENEURO.0230-20.2020 Copyright (C) 2021 Urai et al.

This is an open-access article distributed under the terms of the Creative Commons Attribution 4.0 International license, which permits unrestricted use, distribution and reproduction in any medium provided that the original work is properly attributed.
Laboratory et al., 2020), weekend regimes of traditional water restriction versus free $\mathrm{CA}$ water led to similar weight and learning curves. Free access to $2 \%$ CA water thus allows animals more control over their water intake, without negative effects on behavioral performance.

\section{Materials and Methods}

Experiments were approved by ORBEA Animal Welfare Body at the Champalimaud Center for the Unknown (CCU; number 2016/005) and the Cold Spring Harbor Laboratory (CSHL) Institutional Animal Care and Use Committee (protocol 16-13-10-7, amendment approved 2018-07-28).

\section{Experimental design}

The data from cohort 4 are part of a large, public dataset (described in The International Brain Laboratory et al., 2020) and available at https://data.internationalbrainlab. org. All other experiments were collected separately (although in the case of cohort 3, using the same behavioral task and apparatus as described in The International Brain Laboratory et al., 2020; see also below, Decisionmaking task). COVID-19-related lab shutdowns and following restrictions led to unavoidable disruptions of the course of some experiments and prevented the enlargement of sample sizes.

\section{Cohort 1}

Seventeen mice (11 male and six female wild-type Thy1-GCaMP6, C57BL/6 background, 2-3 months) were single-housed. Each animal's baseline weight was recorded on five consecutive days, while they had free access to plain water in their home-cage. They were then divided into three groups (with males and females randomly assigned), each with its own water regime. The first group $(n=8)$ received measured amounts of water each weekday. This was either $600 \mu \mathrm{l}$, or $40 \mu \mathrm{l}$ of water per gram of body weight as measured right before water administration (range 600-1150 $\mu$ l). On Saturday they received double this amount, while no water was administered on Sundays. The second group $(n=5)$ had access to free water in their home-cage, in which CA $(1 \%$ or $2 \%)$ was dissolved. The last group $(n=4)$ remained on free water in their home-cage and served as a control. No animals showed signs of dehydration, or were dropped from the study.

The home-cage bottles with regular or $2 \%$ CA water were weighed daily, and the weight change used as a proxy for the volume of water consumed. To measure thirst, each animal was placed in a cage with $2 \mathrm{ml}$ of plain water for 5 min on days 16, 24, and 29. For the animals on measured water, these $5 \mathrm{~min}$ replaced their daily measured water administration.

Mice in this study were on average nine weeks old on the first day of the experimental intervention. Expected weight curves were computed from weighings of agematched animals not undergoing fluid restriction, from The Jackson Laboratory (2015). 

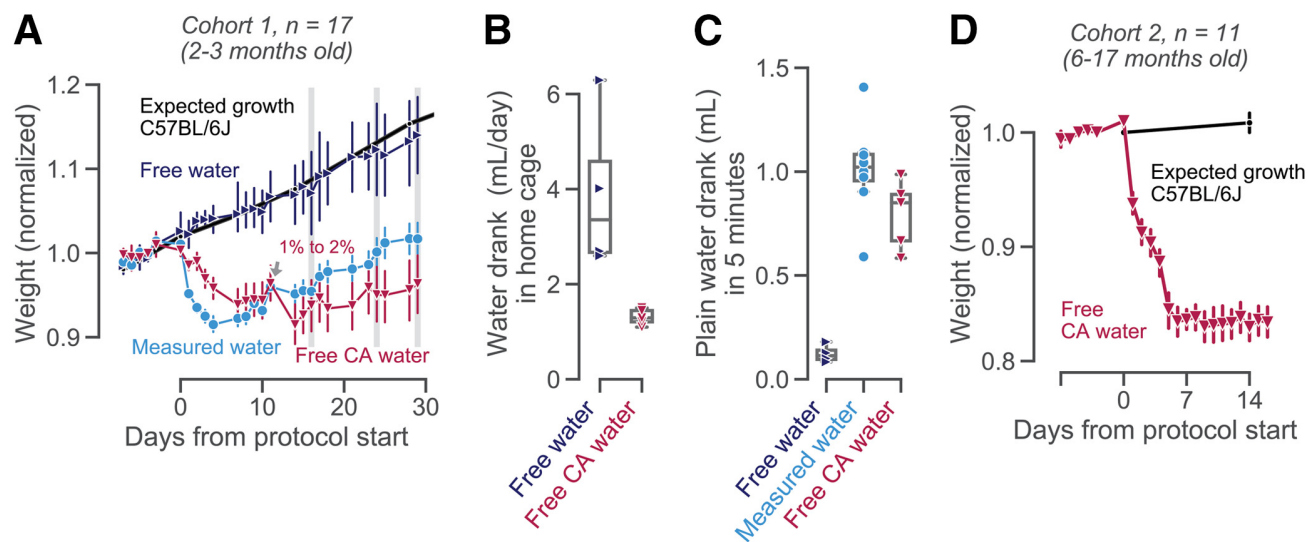

Figure 1. Mice maintain healthy weights and water intake on CA water. A, Average weight (as a fraction of each animal's baseline weight) for 17 young animals, divided into four experimental groups. The age-matched weight curve expected for C57BL/6J mice (The Jackson Laboratory, 2015) on free water is shown as a reference (thick black line). On day 11 (the Friday of the second week after the intervention), the CA group was switched from $1 \%$ to $2 \%$ CA in their home-cage water bottle. Error bars show mean \pm $68 \%$ confidence intervals across animals. $\boldsymbol{B}$, Volume of water drank in the home-cage, estimated by measuring water bottles daily (days 11-29) for the cohorts of mice on free plain (blue) or $2 \%$ CA (red) water. Each data point is the average for one animal; boxplots show median and quartiles. C, Plain water consumed in 5 min of free access, measured on days 16, 24, and 29, indicated with gray shaded bars in $\boldsymbol{A}$. Each data point is the average for one animal; boxplots show median and quartiles. $\boldsymbol{D}$, Weight curves of 11 older animals, before and after switching from free plain water to 2\% CA water. Age-matched expected growth curves for C57BL/6 mice (The Jackson Laboratory, 2018) are shown as a reference in black. See Extended Data Figure 1-1 for growth-corrected and individual weight curves.

\section{Cohort 2}

Twelve mice (five females and seven males, C57BL/6, The Jackson Laboratory, 6-17 months) were co-housed with their siblings. One animal was overweight at the start of the experiment ( $>45 \mathrm{~g}$, body condition score 5 ), and was excluded; we thus show the data for 11 mice in Figure $1 D$. No animals showed signs of dehydration, or were dropped from the study. Each animal's baseline weight was recorded on five consecutive days, while they had free access to plain water in their home-cage. We then switched their home-cage water to $2 \% \mathrm{CA}$, and recorded their daily weight. Laboratory shutdowns because of COVID-19 required us to cease this experiment, preventing a longer-term assessment of the effects of $2 \% \mathrm{CA}$ water on body weight in older mice.

Mice in this study were on average 44 weeks old on the first day of the experimental intervention. Expected weight curves were computed from weighings of age-matched animals not undergoing fluid restriction, from The Jackson Laboratory (2018).

\section{Cohort 3}

Six mice (male C57BL/6, Charles River, 8-18 months) were single-housed. On each weekday, animals performed a decision-making task (full task, as described below). We then varied the liquid regimes, with different types of water administration both during the weekend and weekdays (Fig. 2J). Home-cage bottles were put into the cage on Friday evening and removed on Monday morning, except for the conditions where free water (regular or $2 \% \mathrm{CA}$ ) was also available on weekdays. When no home-cage bottle was available and mice did not earn their minimum required amount of $1 \mathrm{ml} / \mathrm{d}$, they were supplemented with measured water or HydroGel at the end of the day. No animals showed signs of dehydration, or were dropped from the study.

\section{Cohort 4}

We re-analyzed data of mice that completed training on a visual decision-making task (both basic and full tasks, see below) before 23 March 2020. All animals (140 male and female C57BL/6J, experiments performed across seven institutions) started with a week of water restriction, handling and habituation, after which they began training. On weekdays, if they did not earn all their required water in the task, they were supplemented with HydroGel. On weekends, mice received either measured water or HydroGel $(40 \mu \mathrm{l} / \mathrm{g}$ of body weight or $1 \mathrm{ml} / \mathrm{d}$, depending on institutional protocols), or free access to $2 \%$ CA water. See The International Brain Laboratory et al., 2020) for detailed descriptions of the apparatus, handling and husbandry, and automated training protocols. Data are available at https://data.internationalbrainlab.org.

\section{Decision-making task}

Mice learned to perform a visual decision-making task where they detected the presence of a visual grating to their left or right, and reported the perceived location by turning a steering wheel (The International Brain Laboratory et al., 2020). The stimulus moved with the wheel rotation, remaining on the screen until feedback. For each correct response, mice received $1.5 \mu \mathrm{l}$ of $10 \%$ sucrose water. Trial duration was estimated from stimulus onset to the delivery of feedback (either the water reward or a white noise indicating an error). Visual stimuli were presented at variable contrast (0-100\%), allowing us 
Cohort 3, $n=6$ (8-18 months old)

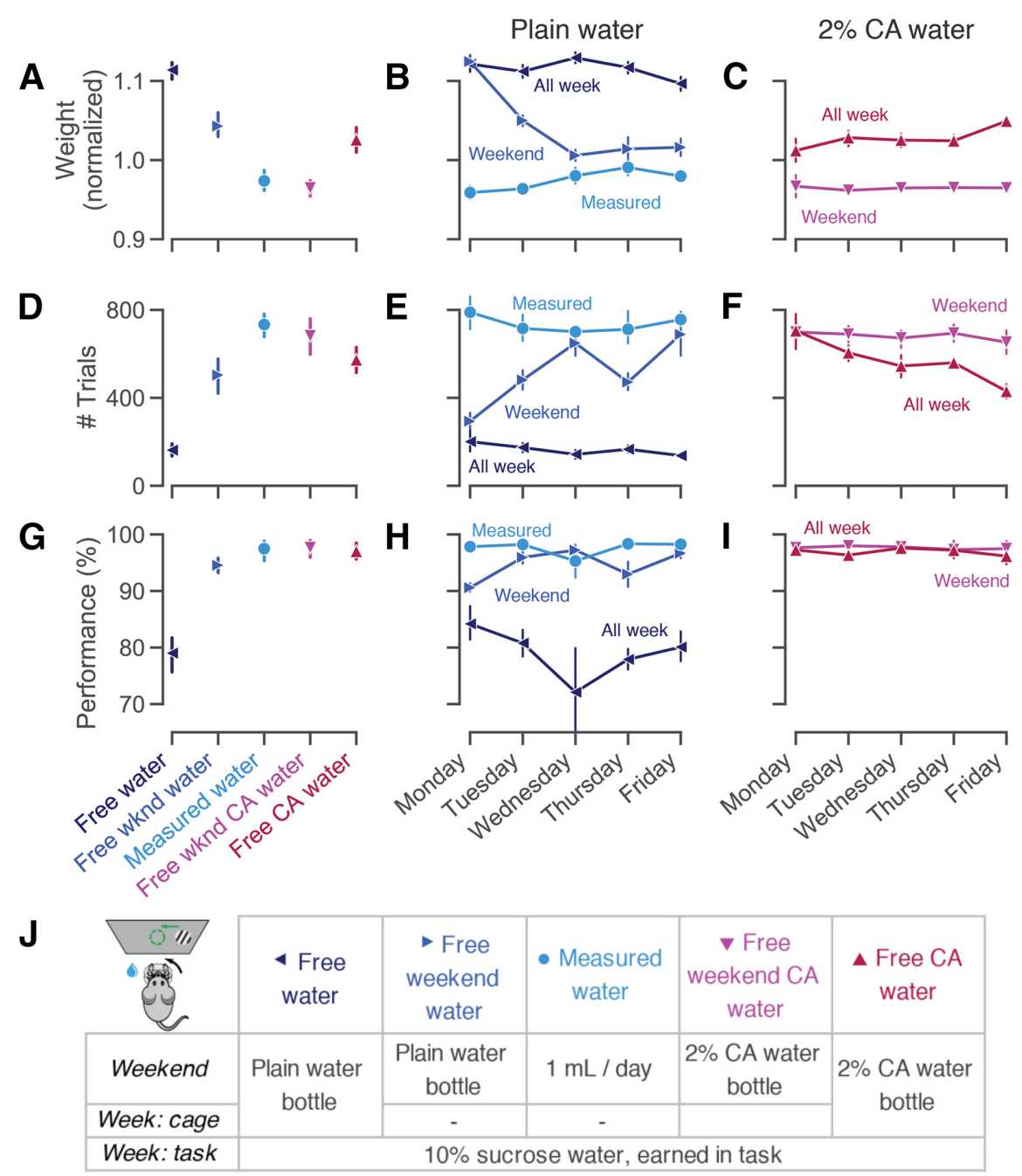

Figure 2. Mice perform many trials, even with free access to CA water. A, Mouse weights per condition, normalized to each animal's average weight over the course of the experiment. $\boldsymbol{B}, \boldsymbol{C}$, Weights as in $\boldsymbol{A}$, per weekday. $\boldsymbol{D}$, Trials performed per condition. $\boldsymbol{E}$, $\boldsymbol{F}$, Trials as in $\boldsymbol{D}$, per weekday. $\mathbf{G}$, Performance on easy trials $(50 \%$ or $100 \%$ visual contrast), per condition. $\boldsymbol{H}, \boldsymbol{I}$, Performance as in $\mathbf{G}$, per weekday. Error bars show mean $\pm 68 \% \mathrm{Cl}$ across animals. $\mathbf{J}$, Schematic of all liquid regimes. Left top corner shows a schematic of the behavioral task. See Extended Data Figure 2-1 for day-by-day data throughout the experiment and Extended Data Figure 2-2 for comparisons with CA dissolved in HydroGel.

to fit psychometric functions to quantify each animal's behavior. The duration of each session was dependent on the engagement of the animal, which was determined through automated criteria (The International Brain Laboratory et al., 2020; see their Fig. 1e and Supplemental Table 3). Early in training, animals were supplemented toward the institutional minimum of $1 \mathrm{ml} / \mathrm{d}$ or $40 \mu \mathrm{l} / \mathrm{g}$ of body after the experimental session. After they became proficient at the behavioral task, they usually performed enough trials to fully earn their water requirement in the rig on weekdays.

A mouse was considered proficient at this basic task once its behavior met a set of prespecified criteria: $>400$ trials performed in each of the last three sessions; accuracy $>80 \%$ correct on easy trials in each of the last three sessions; and on a psychometric function fit on those three sessions combined, a threshold of $<19$, absolute bias of $<16$, and lapses $<0.2$ (The International Brain Laboratory et al., 2020; Appendix 2). Extended Data Figure 3-1E-I shows behavioral data and psychometric function fits from the $3 \mathrm{~d}$ leading up to the animal being trained. The psychometric function has the following form:

$$
\psi(x)=\gamma+(1-\gamma-\lambda) \operatorname{erf}\left(\frac{x-\mu}{\sigma}+1\right) / 2,
$$

where $x$ is the signed visual contrast, $\mu$ is a stimulus-independent bias term, $\sigma$ is the steepness of the psychometric function, and $\gamma$ and $\lambda$ are lapse rates. After reaching task proficiency, animals proceeded to a more complex full task, where they combined visual information with an asymmetric stimulus prior that switched between blocks (The International Brain Laboratory et al., 2020). 
A

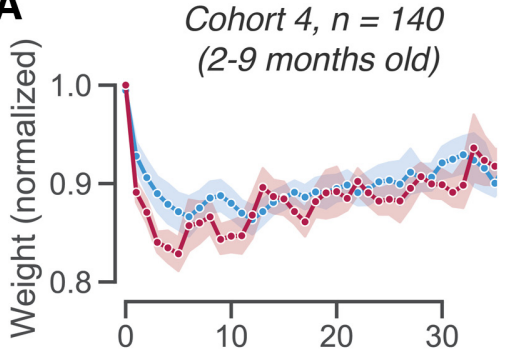

Days from start of water restriction
B

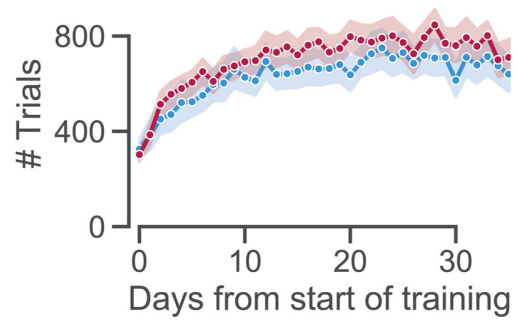

C

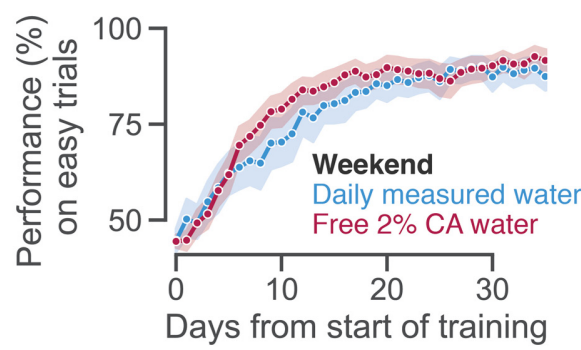

D

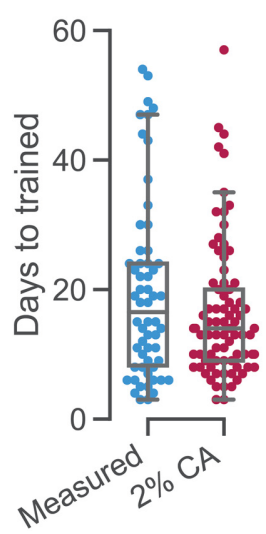

E

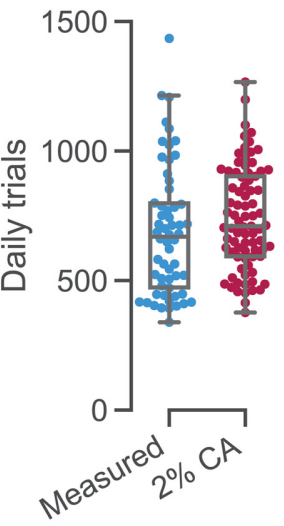

$\mathbf{F}$

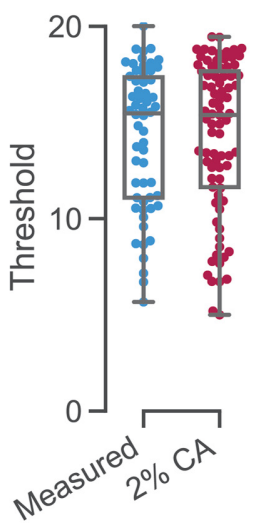

G

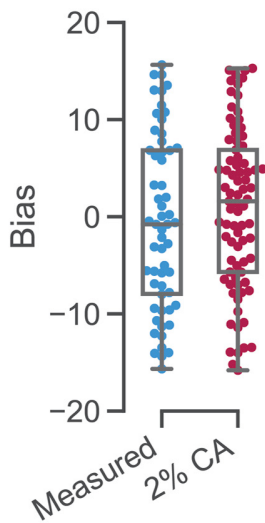

H

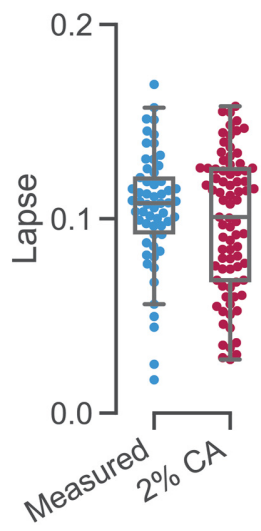

I

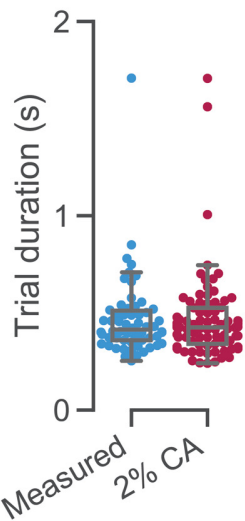

Figure 3. Weekend CA water does not adversely affect learning behavior. $\boldsymbol{A}$, Weight curves (normalized by each animal's free water weight), separately for animals receiving measured water (in accordance with local IACUC protocols) or free $2 \%$ CA water on weekends and other non-training days. $\boldsymbol{B}$, Trial counts in a decision-making task over the course of learning. Training started $\sim 8 \mathrm{~d}$ after the beginning of water restriction. C, Learning curves, showing performance on easy trials with $50 \%$ or $100 \%$ visual contrast. $\boldsymbol{D}-\boldsymbol{I}$, Various measures of learning rates and stable behavior, separated by the weekend regime used in each lab. $\boldsymbol{D}$, Number of days until reaching training criteria. $\boldsymbol{E}-\boldsymbol{I}$, For the three days over which animals passes training criteria, $(\boldsymbol{E})$ average number of trials performed per day, $(\boldsymbol{F})$ threshold from a psychometric function fit, $(\boldsymbol{G})$ choice bias from a psychometric function fit, $(\boldsymbol{H})$ lapse rate from a psychometric function fit, and $(I)$ median trial duration. See Extended Data Figure 3-1 for an examination of sex differences.

\section{CA water preparation}

CA (Sigma-Aldrich) was dissolved into tap water at $1 \%$ or $2 \%$ mass/volume $(\mathrm{m} / \mathrm{v})$. That is, at $2 \% \mathrm{~m} / \mathrm{v}, 2 \mathrm{~g}$ of $\mathrm{CA}$ powder was mixed into $100-\mathrm{ml}$ water and shaken until dissolved. Bottles were replaced with fresh CA water weekly, or when empty.

\section{Data analysis}

Data about each animal (sex, date of birth, lineage), their water intake and weights were logged in the Alyx colony management system (The International Brain Laboratory et al., 2019) and analyzed using DataJoint (Yatsenko et al., 2018). We visualized all data in python, using pandas and seaborn (Waskom et al., 2020). Statistics were done using the Pingouin package (Vallat, 2018).

For psychometric function parameters, as well as learning rates, daily trial counts and trial durations, we report statistics from an independent $t$ test to test the effect of weekend water regime (Fig. 3). The degrees of freedom were corrected for unequal variances using a WelchSatterthwaite correction. The corresponding Bayes factor indicates evidence for the null hypothesis of no difference when $<1$.

\section{Code and data availability}

All code and instructions for reproducing figures and statistics are available at https://github.com/int-brain-lab/ citricAcid.

\section{Results}

\section{Mice maintain health weights and water intake on CA water}

We first confirmed that mice remained healthy and maintained stable weights when given free access to $C A$ water (Fig. 1A). Three subgroups of young animals (cohort 1: total $n=17,2-3$ months old) were given measured amounts of daily water, free plain water, or free CA water in their home-cage. All animals were healthy (as judged by frequent experimenter handling and inspection) and showed no signs of dehydration. Over time, mice with access to free plain water steadily gained weight (Fig. 1A, dark blue), as expected for young C57BL/6J mice with access to free food and water (Fig. $1 A$, thick black line). 
Mice on measured water rapidly lost weight over the first week, after which their weight loss reversed (Fig. 1A, light blue line), matching previous reports (Guo et al., 2014). Mice with access to CA water at a low concentration ( $1 \%$ $\mathrm{m} / \mathrm{v}$ ) did not show adverse health effects nor signs of dehydration, and lost only modest amounts of weight (Fig. $1 A$, red line). These animals also tolerated $\mathrm{CA}$ at a higher concentration of $2 \% \mathrm{~m} / \mathrm{v}$ (Fig. $1 A$ ), in line with findings in rats (Reinagel, 2018). With free access to CA water, animals' weights stabilized after about a week (Fig. 1A, days 11-30).

Since these young animals (on average nine weeks old at the start of the experiment) were still growing, their weights did not solely reflect the water regime. Taking into account this expected weight gain with age, animals on free CA water showed stable weights around $78-95 \%$ (Extended Data Fig. 1-1A,B). We also repeated weight measurements in older mice (Fig. 1D). After switching to free CA water, animals were healthy and maintained stable weights (Fig. 1D). In the second week after introducing $2 \%$ CA water, animals retained on average $83 \%$ (78$91 \%$ ) of their baseline body weight (Extended Data Fig. $1-1 C$ ). While a weight loss of $20 \%$ is considerably larger than for rats with free access to $2 \%$ CA water (who retained $97 \%$ of their baseline body weight, Reinagel, 2018), it is similar to those stable weights obtained under widely used water restriction protocols $(75-85 \%$ after several weeks of water restriction; Guo et al., 2014).

As expected from CA-induced weight loss, animals with access to CA water reduced their fluid intake (Fig. $1 B, C)$. Mice consumed $3.9 \mathrm{ml}$ of plain water per day (range 2.6-6.3 ml; Fig. 1B, blue), but only about one third as much $2 \%$ CA water (1.3 ml, range 1.3-1.5 ml; Fig. $1 B$, red). Importantly, mice with access to free $2 \%$ CA water were still motivated to drink plain water. In 5 min of free access, they drank $0.58-0.99 \mathrm{ml}$ of plain water, similar to animals receiving daily measured water (Fig. 1C). Not only did animals tolerate $2 \%$ CA water well, these data also suggest that free access to CA water might not impede animals' motivation to perform behavioral tasks to earn more palatable liquids.

\section{Mice perform many trials, even with free access to CA water}

In a new cohort of animals (cohort 2: $n=6,8-18$ months) that had been trained on the visual decision-making task (The International Brain Laboratory et al., 2020), we tested how different liquid regimes affected animals' willingness to work for sweetened water rewards (Fig. 2). Animals performed a decision-making task for 45-90 min each weekday and earned a drop of $10 \%$ sucrose water for each correct response. The length of each session was dictated by the automated detection of the animal's engagement, allowing them to work to satiety (The International Brain Laboratory et al., 2020; see their Fig. $1 e$ and Supplemental Table 3). Expert animals usually earned more than their minimum daily water requirement (determined by institutional protocols) in the task and did not require supplemental fluids. In this cohort, we then varied the liquid regime from week to week over several months (Fig. 2J; Extended Data Fig. 2-1).

On weekends and other non-training days, mice are commonly given either daily measured water, or free access to a bottle in their home-cage. When mice drank measured amounts $(1 \mathrm{ml})$ of plain water on weekends (light blue circle), they performed sessions of 730-1230 trials stably over the week (Fig. 2E). This practice is commonly used in laboratories that perform high-throughput mouse training. It is also the most laborious for the experimenter, who needs to provide measured amounts of water each day of the weekend. On the contrary, free home-cage water during the weekend (blue, rightwardpointing triangle) resulted in lower trial counts and worse performance on Mondays and Tuesdays (97-420 trials on Mondays vs $668-1140$ trials on Fridays; $t_{(5)}=-3.951$, $p=0.0109, \mathrm{Bf}_{10}=6.491$; Fig. $2 E, H$; Busse et al., 2011). Under this regime, weights often fluctuated dramatically over the course of the week (weight loss of around $10 \%$ of body weight or $\sim 3 \mathrm{~g}$ between Mondays and Fridays; Fig. $2 B)$, which may have adverse effects on animal health (Rowland, 2007).

Free access to 2\% CA water over the weekend (pink, downward pointing triangle) combines the best of both approaches. Without requiring experimenter intervention on days without training, animals maintained stable weights (Fig. 2C), and consistently performed many trials (Fig. 2F). We could not detect a significant difference in the number of trials performed after a weekend with free $2 \%$ CA water (682 trials/d, range 535-826) versus measured water $\left(733\right.$ trials $/ d$, range $634-817 ; t_{(5)}=0.880, p=$ $0.4193, \mathrm{Bf}_{10}=0.505$; Fig. $2 D$ ). Similarly, high trial yields were achieved by providing CA dissolved in HydroGel cups, a practical alternative in cases where bottled water is difficult to provide (Extended Data Fig. 2-2).

Free CA water yields the most trials when given only on weekends, but can also be kept in the home-cage throughout the week. When free 2\% CA was available continuously (red, upward pointing triangle), the number of trials slightly decreased from Monday to Friday (4351019 on Mondays vs $312-513$ on Fridays; $t_{(5)}=3.002$, $p=0.0300, \mathrm{Bf}_{10}=3.035$; Fig. $2 F$ ). As a result, the average number of trials was slightly lower than when the $2 \% \mathrm{CA}$ water bottle was removed on Monday morning (average 573 vs 682 trials, $t_{(5)}=-2.505, p=0.0542, \mathrm{Bf}_{10}=1.971$; Fig. 2D). While both continuous and weekend-only CA water allow for stable health and fairly high motivation, a regime with continuous $2 \% \mathrm{CA}$ water availability may not consistently yield the high trial numbers required for some experimental purposes.

\section{Weekend CA water does not adversely affect learning behavior}

Since mice maintained high motivation with free CA water on weekends, we implemented this strategy across several laboratories in a large-scale neuroscience collaboration (The International Brain Laboratory et al., 2020). All animals underwent standardized surgeries and training protocols to learn a visual decision-making task (The International Brain Laboratory et al., 2020). Because of 
differences in local animal care arrangements and licenses, two different protocols were used on weekends: four labs (82 mice) used free 2\% CA water, whereas the other five labs (58 mice) gave measured water or HydroGel. This allowed us to investigate the effects of CA water in a large cohort of animals, trained in different laboratories across seven institutions.

Across all labs, and regardless of the weekend water regime, animals successfully learned the task over the course of a few weeks (Fig. 3B,C). Both water regimes also resulted in similar weight curves, with a characteristic rapid drop and subsequent slow weight gain as animals learned to earn sucrose water in the task (Fig. 3A). The number of days needed to complete training (determined as reaching a specified set of behavioral criteria; The International Brain Laboratory et al., 2020; see their Supplemental Table 2), did not vary with weekend water strategy $\left(t_{(101)}=-1.47, p=0.145, \mathrm{Bf}_{10}=0.491\right.$; Fig. $\left.3 D\right)$. Upon training completion, multiple measures of animal behavior were indistinguishable between those labs using measured water versus CA water on weekends: the number of trials performed per day $\left(t_{(104)}=1.33\right.$, $p=0.188, \mathrm{Bf}_{10}=0.410$; Fig. $\left.3 E\right)$, visual threshold $\left(t_{(128)}=\right.$ $0.10, p=0.919, \mathrm{Bf}_{10}=0.185$; Fig. $3 F$ ), choice bias $\left(t_{(116)}=1.05, p=0.297, \mathrm{Bf}_{10}=0.304 ;\right.$ Fig. 3G), lapse rate $\left(t_{(133)}=-1.36, p=0.175, \mathrm{Bf}_{10}=0.429\right.$; Fig. $\left.3 H\right)$, and median trial duration $\left(t_{(128)}=-0.03, p=0.978, \mathrm{Bf}_{10}=0.184\right.$; Fig. 3/). These patterns were similar for both male and female mice (Extended Data Fig. 3-1). This suggests that CA water is a reliable alternative to water restriction for achieving high quality mouse behavior.

\section{Discussion}

High-throughput, reliable behavioral training is a key requirement for the use of mice in behavioral and systems neuroscience. Here, we have shown that free access to CA water is well tolerated, and motivates mice to perform many trials of a decision-making task in which they earn sugar water. We thus consider CA water a promising alternative to water restriction for some experimental regimes.

In contrast to commonly used water restriction regimes, free access to CA water allows animals full control over the amount and timing of their water intake. Home-cage access to food and CA water also enables animals to eat and drink simultaneously, which benefits their metabolism and nutrition (Fertig and Edmonds, 1969; Toth and Gardiner, 2000). In some institutions, drinking water is acidified to reduce bacterial growth (Reinagel, 2018). Since $2 \%$ CA water has $\mathrm{pH} 2.07$, it may convey antibacterial properties that benefit animal welfare.

Beyond extending previous experiments with CA water from rats (Watson et al., 1986; Reinagel, 2018) to mice, we here propose a new variant that maintains high trial yields. Young rats with continuous access to $2 \%$ CA water perform only around $68 \%$ of their water-restricted trial counts during daily sessions (84\% for live-in behavioral testing; Reinagel, 2018). Similarly, when CA water was available throughout the week, mice performed around $22 \%$ fewer trials compared with usual conditions (no home-cage water available on testing days, measured water on weekends). Such continuous CA accessibility may thus be suitable if high trial yields are not needed, or if the baseline trial count is sufficiently high that an acceptable number of trials can be collected. As an alternative, we here propose that providing CA water only on weekends and other non-training days balances animal welfare with ease of use and behavioral throughput.

While we have achieved a satisfactory balance between water intake and trial counts using a concentration of $2 \%$ $\mathrm{CA}(\mathrm{m} / \mathrm{v})$, this concentration could be further fine-tuned based on the details of each experimental setup. Trial yield may also depend on individual animal's taste perception (which likely varies between species and strains), task design (e.g., head-fixed vs freely moving), and local factors such as food or environmental humidity. For instance, if only CA water is available in the home-cage, sweetened task water likely further increases animals' willingness to work. The strategy of placing animals on free CA water over the weekend may not be viable for all experiments: for example, studies investigating differences in reward and taste processing, or those requiring precise tracking of individual animal's fluid intake.

Beyond benefits to animal welfare, eliminating water restriction also benefits the quality and throughput of behavioral experiments. It removes the need to give animals supplemental fluids on days when they do not train (e.g., weekends) or days when they do not earn their water requirements in the task (as often happens early in training). Such supplements are often beyond the capacities of routine animal care procedures, and require scientific personnel's daily attention and record-keeping. Besides being highly labor-intensive, water supplements carry a risk of human error in the timing and precise delivery of required water amounts, as well as the distribution of water between co-housed mice. Unexpected absence of experimental personnel usually requires animals to be placed on free plain water, which can impede their progress in behavioral training for many days or weeks afterward.

Free access to CA water provides a relatively nonlabor-intensive, low-error option for keeping animals healthy without hindering behavioral training progress. This suggests that CA water can serve as a reliable and standardized strategy to achieve high quality task behavior, further facilitating the use of mice in high-throughput behavioral studies.

\section{References}

Abbott A (2010) Neuroscience: the rat pack. Nature 465:282-283.

Busse L, Ayaz A, Dhruv NT, Katzner S, Saleem AB, Schölvinck ML, Zaharia AD, Carandini M (2011) The detection of visual contrast in the behaving mouse. J Neurosci 31:11351-11361.

Carandini M, Churchland AK (2013) Probing perceptual decisions in rodents. Nat Neurosci 16:824-831.

Fertig DS, Edmonds VW (1969) The physiology of the house mouse. Sci Am 221:103-113.

Goltstein PM, Reinert S, Glas A, Bonhoeffer T, Hübener M (2018) Food and water restriction lead to differential learning behaviors in a head-fixed two-choice visual discrimination task for mice. PLoS One 13:e0204066. 
Guo ZV, Hires SA, Li N, O'Connor DH, Komiyama T, Ophir E, Huber D, Bonardi C, Morandell K, Gutnisky D, Peron S, Xu NI, Cox J, Svoboda K (2014) Procedures for behavioral experiments in headfixed mice. PLoS One 9:e88678.

Reinagel P (2018) Training rats using water rewards without water restriction. Front Behav Neurosci 12:84.

Rowland NE (2007) Food or fluid restriction in common laboratory animals: balancing welfare considerations with scientific inquiry. Comp Med 57:149-160.

Skinner BF (1936) Thirst as an arbitrary drive. J Gen Psychol 15:205210.

The International Brain Laboratory; Aguillon-Rodriguez V, Angelaki DE, Bayer HM, BonacchiN, Carandini M, Cazettes F, Chapuis GA, Churchland AK, Dan Y, Dewitt EE, Faulkner M, Forrest H, Haetzel LM, Hausser M, Hofer SB, Hu F, Khanal A, Krasniak CS, Laranjeira I, et al. (2020) A standardized and reproducible method to measure decision-making in mice. bioRxiv. doi: https://doi.org/10.1101/ 2020.01.17.909838.

The International Brain Laboratory; Bonacchi N, Chapuis G, Churchland AK, Harris KD, Rossant C, Sasaki M, Shen S, Steinmetz NA, Walker EY, Winter O, Wells M (2019) Data architecture and visualization for a large-scale neuroscience collaboration. bioRxiv. doi: https://doi.org/10.1101/827873.
The Jackson Laboratory (2015) Body weight information for C57BL/6J (000664). Available at https://www.jax.org/jax-mice-and-services/ strain-data-sheet-pages/body-weight-chart-000664.

The Jackson Laboratory (2018) Body weight information for aged C57BL/ 6J (000664). Available at https://www.jax.org/jax-mice-and-services/ strain-data-sheet-pages/body-weight-chart-aged-b6.

Toth LA, Gardiner TW (2000) Food and water restriction protocols: physiological and behavioral considerations. Contemp Top Lab Anim Sci 39:9-17.

Vallat R (2018) Pingouin: statistics in Python. J Open Source Softw 3:1026.

Waskom M, Botvinnik O, Ostblom J, Lukauskas S, Hobson P, MaozGelbart, Gemperline DC, Augspurger T, Halchenko Y, Cole JB, Warmenhoven J, de Ruiter J, Pye C, Hoyer S, Vanderplas J, Villalba S, Kunter G, Quintero E, Bachant P, Martin M, et al. (2020) Seaborn. Zenodo. Available at https://zenodo.org/ record/3629446.

Watson PJ, Beatey S, Wagner F, Stahl T (1986) Water adulteration with citric acid: effects on drinking and responsivity to regulatory challenges. Physiol Behav 36:329-338.

Yatsenko D, Walker EY, Tolias AS (2018) DataJoint: a simpler relational data model. arXiv: 1807.11104. 